\title{
COMUNIDADES DE PARASITÓIDES DE Leucoptera coffeella (Guérin-Mèneville \& Perrottet, 1842) (Lepidoptera: Lyonetiidae) EM CAFEEIROS NAS REGIOEES OESTE E SUDOESTE DA BAHIA
}

\author{
Parasitoid communities of Leucoptera coffeella (Guérin-Mèneville \& Perrottet, 1842) \\ (Lepidoptera: Lyonetiidae) in coffee plants in the western and \\ southwestern regions of Bahia state, Brazil
}

\author{
Thiago Lima Melo ${ }^{1}$, Maria Aparecida Castellani ${ }^{2}$, Maria de Lordes do Nascimento ${ }^{3}$, \\ Ayres de Oliveira Menezes Junior ${ }^{4}$, Gabriel Fernandes Pinto Ferreira ${ }^{5}$, Odair Lacerda Lemos ${ }^{6}$
}

\begin{abstract}
RESUMO
Objetivou-se conhecer a diversidade e a estrutura das comunidades de parasitóides de Leucoptera coffeella (Guérin-Mèneville \& Perrottet, 1842) nas regiões Oeste, município de Luiz Eduardo Magalhães, e, Sudoeste, município de Vitória da Conquista, do Estado da Bahia. Os estudos foram desenvolvidos nos anos de 2002 e 2003, por meio de coletas mensais de folhas do quarto par, de ramos nos três estratos da planta e caídas no solo, totalizando 1600 folhas por região e período de coleta, coletando-se minas contendo pupas de parasitóides e crisálidas. O material foi mantido no Laboratório de Entomologia da Universidade Estadual do Sudoeste da Bahia, em temperatura ambiente, visando à emergência de adultos. A estrutura das comunidades foi avaliada por meio dos índices faunísticos freqüência, constância, dominância e diversidade. Foram identificadas seis espécies de parasitóides pertencentes à Braconidade e Eulophidae, registrando-se os parasitóides Cirrospilus neotropicus (Diez \& Fidalgo, 2003); Closteroscerus coffeellae (Ihering, 1914); Horismenus aeneicollis (Ashmead, 1904); Neochrysocharis coffeae (Ihering, 1914); Stiropius sp.1 e Stiropius sp.2, associados ao bicho-mineiro do cafeeiro. Há diferenças na estrutura das comunidades de parasitóides em função das regiões estudadas, sendo que na Região Oeste a espécie predominante foi N. coffeae, enquanto que na região Sudoeste, H. aeneicollis e Stiropius sp.1 foram as predominantes.
\end{abstract}

Termos para indexação: Análise faunística, microhimenóptero, café.

\section{ABSTRACT}

The aim of this work was to know the diversity and the structure of parasitoid communities of Leucoptera coffeella (GuérinMèneville \& Perrottet, 1842) in the West, municipality of Luiz Eduardo Magalhães, and in the Southwest, municipality of Vitória da Conquista, of the State of Bahia, Brazil. The studies were developed in 2002 and 2003, by monthly samplings of leaves from the fourth pair, of branches in the three plant strata and those fallen on the ground, with a total of 1600 leaves per region and sampling period, and by collecting mines containing pupae of parasitoids and chrysalis. The material was kept in the Entomology Laboratory of the State University of Southwestern Bahia (Universidade Estadual do Sudoeste da Bahia - UESB), under ambient temperature, for the emergency of adults. The structure of communities was assessed by means of the faunistic indexes, frequency, constancy, dominance and diversity. Six parasitoid species belonging to Braconidade and Eulophidae were identified, namely parasitoids Cirrospilus neotropicus (Diez \& Fidalgo, 2003); Closteroscerus coffeellae (Ihering, 1914); Horismenus aeneicollis (Ashmead, 1904); Neochrysocharis coffeae (Ihering, 1914); Stiropius sp.1 and Stiropius sp.2 associated to plant coffee leaf miner. There are differences in the structure of parasitoid communities according to the studied regions, being given that in the Western Region the predominant species was N. coffeae, while in the Southwestern Region, H. aeneicollis and Stiropius sp.1 were the predominant ones.

Index terms: Faunistic analysis, microhymenoptera, coffee.

\section{(Recebido em 9 de março de 2006 e aprovado em 8 de novembro de 2006)}

\section{INTRODUÇÃO}

A cafeicultura na Bahia é consolidada nas regiões do Planalto (Vitória da Conquista, Chapada Diamantina, Jequié, Santa Inês, Itiruçu, Brejões), do Cerrado (Oeste da Bahia) e na faixa Litorânea (Sul, Baixo Sul e Extremo Sul), caracterizadas por apresentarem condições edafoclimáticas e ecológicas distintas e sistemas de cultivo diferenciados.

\footnotetext{
Mestre em Agronomia - Departamento Fitotecnia e Zootecnia/DFZ - Universidade Estadual do Sudoeste da Bahia/UESB - Laboratório de Entomologia Cx. P. 95 - 45083-900 - Vitória da Conquista, BA - thiagolimelo@yahoo.com.br

${ }^{2}$ Doutora, Professora Titular - Departamento Fitotecnia e Zootecnia/DFZ - Universidade Estadual do Sudoeste da Bahia/UESB - Laboratório de Entomologia - Cx. P. 95 - 45083-900 - Vitória da Conquista, BA - castellani@uesb.br

${ }^{3}$ Doutora, Pesquisadora - Departamento Fitotecnia e Zootecnia/DFZ - Universidade Estadual do Sudoeste da Bahia/UESB - Laboratório de Entomologia Cx. P. 95 - 45083-900 Vitória da Conquista, BA - mlnmalu@uesb.br - Bolsista PRODOC/FAPESB

${ }^{4}$ Doutor, Professor Adjunto - Departamento de Agronomia - Universidade Estadual de Londrina/UEL - Campus Universitário - Cx. P. 6001 - $86051-990$ Londrina, PR - ayres@uel.br

${ }^{5}$ Graduando em Agronomia - Departamento Fitotecnia e Zootecnia/DFZ - Universidade Estadual do Sudoeste da Bahia/UESB - Laboratório de Entomologia - Cx. P. 95 - 45083-900 - Vitória da Conquista, BA -gabrielfpf@hotmail.com - Bolsista PIBIC/CNPq

${ }^{6}$ Mestre em Agronomia - Departamento Fitotecnia e Zootecnia/DFZ - Universidade Estadual do Sudoeste da Bahia/UESB - Laboratório de Entomologia Cx. P. 95 - 45083-900 - Vitória da Conquista, BA - odairlacerda@hotmail.com
} 
O cafeeiro hospeda diversas espécies de artrópodes, algumas das quais assumem o "status" de praga, destacando-se a broca-do-café, Hypothenemus hampei (Ferrari, 1867) (Coleoptera: Scolytidae) e o bichomineiro-do-cafeeiro, Leucoptera coffeella (GuérinMèneville \& Perrottet, 1842), como pragas-chave na maioria dos cultivos dessa planta no Brasil (REIS JÚNIOR, 1999).

A dinâmica populacional do bicho-mineiro-docafeeiro é variável em função das regiões de cultivo, devido a fatores bióticos e abióticos que atuam no agroecossistema cafeeiro. Nesse contexto, predadores e parasitóides são importantes organismos que contribuem na regulação das populações do bicho-mineiro (SOUZA \& REIS, 2000).

Os himenópteros parasitóides são elementos importantes por seu papel no controle da população de outros insetos que interferem, direta ou indiretamente e de forma ainda não quantificada, nas cadeias tróficas de grande parte dos agroecossistemas. Devido à sua capacidade em contribuírem de forma efetiva como reguladores das populações de muitos insetos considerados pragas, algumas espécies de himenópteros parasitóides podem ser utilizadas com sucesso em programas de controle biológico e no manejo integrado de pragas (PERIOTO et al., 2004).

Nas regiões Oeste e Sudoeste da Bahia, o bichomineiro-do-cafeeiro ocorre durante o ano todo, de forma sistemática, geralmente exigindo a adoção do controle químico, especialmente na região Oeste, onde as condições são mais favoráveis ao desenvolvimento da praga. $\mathrm{O}$ uso do controle químico como principal estratégia de manejo do bicho-mineiro tem provocado impacto significativo sobre a fauna benéfica associada (PARRA et al., 1981).

Cerca de 18 espécies de parasitóides, pertencentes às famílias Braconidae e Eulophidae (Hymenoptera) encontram-se associadas ao bicho-mineiro-do-cafeeiro na América (GALLARDO-COVAS, 1992). No Brasil, alguns pesquisadores têm relatado o papel dos inimigos naturais do bicho-mineiro-do-cafeeiro nos Estados do Paraná (CARNEIRO FILHO \& GUIMARÃES, 1984), São Paulo (ATIQUE et al., 1981; MENDES, 1940; PARRA et al., 1977; TOZATI \& GRAVENA, 1988) e Minas Gerais (AVILÉS, 1991; SOUZA, 1979).

Atualmente, os princípios de produção integrada estão sendo adotados como modelo de desenvolvimento sustentável para a cafeicultura, e o manejo integrado de pragas como base para decisões sobre proteção de plantas, visando à proteção da diversidade biológica, minimização de custos externos, impactos indesejados e estabilidade dos agroecossistemas (RAIJ, 2003). Nesse contexto, conhecimento a cerca dos níveis populacionais das pragaschave, da diversidade de parasitóides e dos índices de parasitismo natural são fundamentais para a tomada de decisão na adoção de estratégias de manejo.

Em virtude da carência de informações sobre inimigos naturais do bicho-mineiro-do-cafeeiro para as regiões Oeste e Sudoeste da Bahia, desenvolveu-se o presente trabalho, cujo objetivo foi conhecer a diversidade e estrutura das comunidades desses parasitóides.

\section{MATERIAL E MÉTODOS}

Os estudos foram desenvolvidos na Fazenda Agronol, latitude $11^{\circ} 57^{\prime} \mathrm{S}$, longitude $45^{\circ} 43^{\prime} \mathrm{W}$, altitude de 780 m, localizada no município de Luiz Eduardo Magalhães, no Oeste da Bahia, no período de novembro de 2002 a outubro de 2003. O clima é tipo Aw, quente, com estação seca acentuada, temperatura do mês mais frio de $22,2^{\circ} \mathrm{C}$ e precipitação anual média de $1.100 \mathrm{~mm}$. Nessa propriedade os cafeeiros eram da cultivar Catuaí Vermelho IAC-144, irrigado por sistema de pivô central concêntrico, adensado, com espaçamento de 3,75 metros entre linhas e 0,50 metros entre plantas, com aproximadamente 4 anos de idade.

Na Região Sudoeste da Bahia, os estudos foram realizados na Fazenda Natureza, latitude $15^{\circ} 00^{\prime}$ 'S, longitude $47^{\circ} 43^{\prime} \mathrm{W}$, altitude de $883 \mathrm{~m}$, localizada no Povoado de Capinal, município de Vitória da Conquista, no período de novembro de 2002 a dezembro de 2003. Segundo a classificação de Köopen, o clima da região é do tipo Am, quente, tropical úmido, com chuvas do tipo moções, e, Aw, quente, com estação seca acentuada, temperatura do mês mais frio de $18,0^{\circ} \mathrm{C}$ e precipitação anual média de $900 \mathrm{~mm}$. O cafezal selecionado era de sequeiro, formado pela cultivar Catuaí Amarelo, com idade de 25 anos, com espaçamento de 4,0 metros entre linhas e 1,5 metros entre plantas.

As amostragens foram realizadas a cada 30 dias, coletando-se o quarto par de folhas de ramos localizados no terço inferior, médio e superior da planta e, também, folhas caídas no solo contendo minas e/ou crisálidas. Foram coletadas dez folhas de cada estrato e do solo em 40 plantas, totalizando 1.600 folhas em cada área de estudo e período de amostragem. As folhas coletadas foram acondicionadas em sacos de papel, etiquetados, colocadas em caixa de isopor e transportadas para o laboratório de Entomologia da Universidade Estadual do Sudoeste da Bahia. Folhas contendo crisálidas do bicho-mineiro e pupas de parasitóides foram acondicionadas em recipientes de plástico transparente, cobertos com "voil", e mantidos em temperatura ambiente visando à emergência dos parasitóides. Estes foram fixados em álcool 70\%, etiquetados e encaminhados para identificação. 
A suficiência amostral foi verificada por meio da Curva do Coletor, construída a partir das unidades amostrais e número acumulativo de espécies amostradas (CAIN, 1938, citado por SILVA \& LOECK, 1999). A distribuição dos pontos ajustou-se uma equação logarítmica.

A caracterização das comunidades de parasitóides do bicho-mineiro foi feita por meio dos índices faunísticos. A freqüência relativa, que representa a participação porcentual do número de indivíduos da espécie em relação ao total de indivíduos coletados, foi calculada utilizando-se a fórmula: $\mathrm{f}=\mathrm{n} / \mathrm{N}$ x 100 , em que $f$ é a porcentagem de freqüência, $n$ é o número de indivíduos de cada espécie e $N$ o número total de indivíduos obtidos em cada coleta. A constância foi obtida através da porcentagem de ocorrência das espécies nas coletas, baseando-se na fórmula $\mathrm{C}=\mathrm{P} / \mathrm{N}$ x 100 , em que $C$ é a porcentagem de constância, $P$ é o número de coleta e $N$ o número total de coletas (SILVEIRA NETO et al., 1976). Posteriormente, as espécies foram separadas por categorias, segundo a classificação de Bodenheimer (1955), citado por Silveira Neto et al. (1976), em: espécies constantes (W), presentes em mais de 50\% das coletas; espécies acessórias (Y), presentes em 25 a $50 \%$ das coletas e espécies acidentais (Z), presentes em menos de $25 \%$ das coletas. Uma espécie é considerada dominante quando apresenta uma frequiência superior a $1 / \mathrm{S}$, em que, $S$ é o número total de espécies na comunidade. A riqueza $(S)$ foi obtida pelo número total de espécies observadas na comunidade. A diversidade foi medida pelo índice de diversidade $(\alpha)$, que é a relação entre o número de espécies (S) e o número de indivíduos de uma comunidade $(\mathrm{N})$, dada pela fórmula: $=\alpha(\mathrm{S}-1) / \mathrm{LN}$ (SILVEIRA NETO et al., 1976).

\section{RESULTADOS E DISCUSSÃO}

Para as condições do cafezal localizado no município de Vitória da Conquista, a Curva do Coletor estabilizou-se a partir da terceira coleta, o mesmo não ocorrendo para a outra área estudada, possivelmente em função do emprego de inseticidas para o controle do bicho-mineiro-do- cafeeiro no ano anterior ao início do experimento (Figura 1).

Considerando-se as duas áreas de estudo, foram coletados 701 himenópteros pertencentes às famílias Eulophidae e Braconidae, distribuídos em cinco gêneros e seis espécies: Cirrospilus neotropicus (Diez \& Fidalgo, 2003) (Eulophidae); Closterocerus coffeellae (Ihering, 1914) (Eulophidae); Horismenus aeneicollis (Ashmead, 1904) (Eulophidae); Neochrysocharis coffeae (Ihering, 1914) (Eulophidae); Stiropius sp.1 (Braconidae) e Stiropius sp.2 (Braconidae).

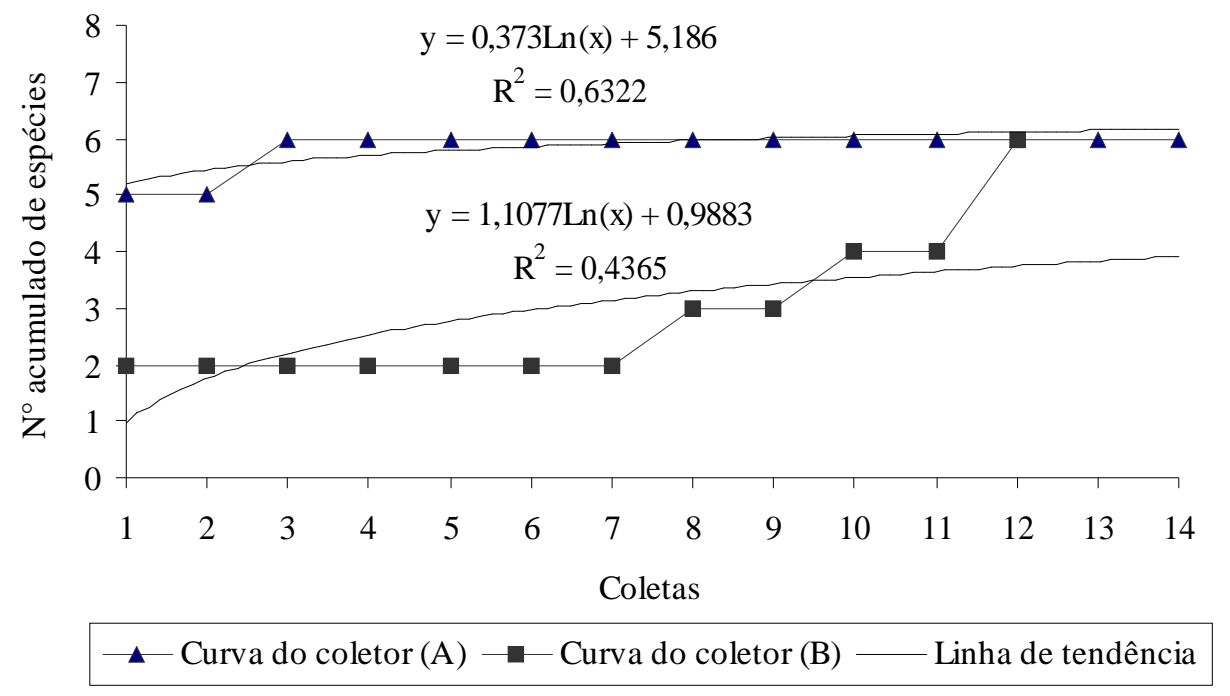

FIGURA 1 - Curva do coletor do número acumulado de espécies de parasitóides e estimativa do número de espécies em função do número de coletas na fazenda Agronol, Vitória da Conquista-BA, no período 11/2002 a 12/2003 (A) e fazenda Natureza, Luiz Eduardo Magalhães-BA, no período de 11/2002 a 10/2003 (B). 
Para as condições do cafezal situado no município de Luiz Eduardo Magalhães, foram coletados 266 indivíduos, inferior ao total obtido para Vitória da Conquista, porém, com participação expressiva dos eulofídeos $(99,24 \%)$. O parasitóide $N$. coffeeae foi a única espécie considerada predominante, apresentando frequiência superior às demais espécies, classificando-se como constante e dominante. Quanto à $C$. coffeellae, apesar de ter ocorrido em baixa freqüência, também foi constante, estando presente em mais de $50,0 \%$ das coletas (Tabela 1).

Para as condições da cultura em Vitória da Conquista, foram coletados 435 indivíduos, com maior participação de eulofídeos $(62,8 \%)$ em relação aos braconídeos $(37,2 \%)$. Os microhimenópteros H. aeneicollis, Stiropius sp. 1 e $N$. coffeae foram predominantes, sendo considerados mais freqüentes, constantes e dominantes. Com exceção de $C$. neotropicus, que foi acessória, todas foram constantes (Tabela 1). A riqueza foi a mesma para as duas regiões e o índice de diversidade semelhante. No entanto, ressalta-se que, para as condições encontradas na lavoura em Luiz Eduardo Magalhães, N. coffeae foi a única espécie predominante, enquanto que os braconídeos Stiropius sp.1 e Stiropius sp. 2 foram considerados constantes para Vitória da Conquista. Além das diferenças climáticas entre as regiões, os cultivos da Região Oeste são altamente tecnificados, com uso intensivo da irrigação, fertilizantes e inseticidas, podendo-se levantar a hipótese de que $N$. coffeae tenha se adaptado às condições de clima e de manejo e possivelmente ser considerada uma espécie de forte valência ecológica e com possibilidade de maior distribuição entre habitats diferentes (SILVEIRA NETO et al., 1976).

Na região de Campinas, Mendes (1940) relatou a ocorrência de Tetrastichus sp., C. coffeellae e Orgilus sp., sendo esse o mais abundante $(75,6 \%)$ do total de parasitóides coletados.

Em algumas regiões do Estado de São Paulo, Parra et al. (1977) coletaram nove espécies de parasitóides, sendo três de braconídeos, Stiropius letifer (Mann, 1872); Orgilus punctatus (Beyr, 1959) e Mirax sp. e seis de eulofideos, $H$. aeneicollis; Horismenus sp.; Tetrastichus sp.; $C$. coffeellae; N. coffeae e Cirrospilus sp.. Igualmente para São Paulo, Atique et al. (1981) constataram que a família Braconidae representou $47 \%$ dos parasitóides em Jaú, sendo que $S$. letifer foi a mais abundante.

TABELA 1 - Análise faunística das espécies de parasitóides de Leucoptera coffeella, municípios de Luiz Eduardo Magalhães e Vitória da Conquista , no Estado da Bahia, 2002/2003.

\begin{tabular}{|c|c|c|c|c|c|c|c|c|}
\hline \multirow{2}{*}{$\overbrace{\text { Espécie }}^{\text {Local }}$} & \multicolumn{4}{|c|}{ Luiz E. Magalhães } & \multicolumn{4}{|c|}{ Vitória da Conquista } \\
\hline & $\mathbf{N}$ & $\mathbf{F}$ & $\mathbf{C}$ & D & $\mathbf{N}$ & $\mathbf{F}$ & $\mathbf{C}$ & D \\
\hline Neochrysocharis coffeae & 201 & 75,6 & $\mathrm{~W}$ & $\mathrm{~d}$ & 78 & 17,9 & $\mathrm{~W}$ & $\mathrm{~d}$ \\
\hline Closterocerus coffeellae & 26 & 9,8 & $\mathrm{~W}$ & nd & 69 & 15,9 & $\mathrm{~W}$ & nd \\
\hline Stiropius sp.1 & 1 & 0,4 & $\mathrm{Z}$ & nd & 126 & 29,0 & $\mathrm{~W}$ & $\mathrm{~d}$ \\
\hline Stiropius sp.2 & 1 & 0,4 & $\mathrm{Z}$ & nd & 36 & 8,3 & $\mathrm{~W}$ & nd \\
\hline Horismenus aeneicollis & 27 & 10,2 & $\mathrm{Z}$ & nd & 115 & 26,4 & $\mathrm{~W}$ & $\mathrm{~d}$ \\
\hline Cirrospilus neotropicus & 10 & 3,8 & $\mathrm{Y}$ & nd & 11 & 2,5 & $\mathrm{Y}$ & nd \\
\hline Total & 266 & 100,0 & - & - & 435 & 100 & - & - \\
\hline Porcentagem & \multicolumn{4}{|c|}{37,95} & \multicolumn{4}{|c|}{62,05} \\
\hline $\mathrm{R}$ & \multicolumn{4}{|c|}{6} & \multicolumn{4}{|c|}{6} \\
\hline ID & \multicolumn{4}{|c|}{0,89} & \multicolumn{4}{|c|}{0,82} \\
\hline
\end{tabular}

$\mathrm{N}$ : número total de indivíduos $(\mathrm{N}) ; \mathrm{F}$ : freqüência relativa; R: riqueza; C: constância, sendo constante $(\mathrm{W})$, acessória $(\mathrm{Y})$ e acidental (Z); D: dominância, sendo dominante (d) e não dominante (nd). 
Para Minas Gerais, Souza (1979) relacionou seis espécies de parasitóides do bicho-mineiro, referindo-se a C. coffeellae; Proacrias sp.; S. letifer e Horismenus sp., como principais, apresentado ampla distribuição no Estado e também maior abundância. Ainda nesse Estado, Avilés (1991) coletou os parasitóides Horismenus cupreus (Ashmead, 1894); Mirax insulares (Muesebeck, 1937) e Elachertus sp. pela primeira vez no Brasil, na região de Viçosa. Foram coletados 560 indivíduos pertencentes a nove espécies, sendo $S$. letifer; $N$. coffeae; $M$. insularis; C. coffeellae e H. cupreus, as mais abundantes.

De modo geral, a riqueza de espécies de parasitóides para os dois municípios no Estado da Bahia foi baixa em relação a outros estados brasileiros, especialmente Minas Gerais. As diferenças na estrutura das comunidades parecem variar em função das diversas regiões cafeeiras do Brasil.

Carneiro Filho \& Guimarães (1984), em trabalho realizado em três regiões cafeeiras do Estado do Paraná, classificadas como quente $\left(22 / 23^{\circ} \mathrm{C}\right)$, intermediária $(20 /$ $\left.22^{\circ} \mathrm{C}\right)$ e fria $\left(19 / 20^{\circ} \mathrm{C}\right)$, obtiveram taxas diferenciadas de parasitismo, sendo maior na região intermediária, e predominância de diferentes espécies de parasitóides. Na região quente, onde o parasitismo foi de 25,60\%, Mirax sp. teve uma participação de $11,06 \%$ e $N$. coffeae de 7,02\%. Na região intermediária, o parasitismo alcançou $44,71 \%$, destacando-se Mirax sp. (16,61\%) N. coffeae (7,68\%), O. punctatus $(7,02 \%)$, S. letifer $(7,37 \%)$ e C. coffeellae $(3,9 \%)$. $\mathrm{Na}$ região mais fria, o parasitismo foi menor $(19,82 \%)$, onde se destacaram $O$. punctatus $(8,37 \%)$, Mirax sp. (3,76\%), $N$. coffeae $(4,04 \%)$ e $S$. letifer $(2,44 \%)$. Esses resultados evidenciam a adaptabilidade de algumas espécies de parasitóides, como N. coffeae e Mirax sp., que ocorreram nas três regiões do Paraná.

No Estado da Bahia, Melo (2005) constatou menor taxa de parasitismo do bicho-mineiro (0 a 9,33\%) no município de Luiz Eduardo Magalhães, região mais quente, em relação à Vitória da Conquista $(0,27$ a 19,36\%), considerada uma região mais fria.

Em relação à influência da posição das folhas (estratos da planta e solo) sobre a abundância de parasitóides (Tabela 2), verificou-se, que o número de espécimes foi maior no estrato superior, para as condições da lavoura localizada em Luiz Eduardo Magalhães, onde houve predominância de $N$. coffeae, um eulofídeo que, provavelmente, parasita lagartas nos estádios iniciais do desenvolvimento. As folhas do estrato inferior apresentaram maior número de parasitóides para as

TABELA 2 - Número total de adultos de parasitóides de Leucoptera coffeella, em função da espécie e localização das folhas coletadas, nos municípios de Luiz Eduardo Magalhães e Vitória da Conquista no Estado da Bahia, 2002/ 2003.

\begin{tabular}{|c|c|c|c|c|c|c|c|c|c|c|c|c|c|c|c|c|}
\hline \multirow{4}{*}{ Espécie } & \multicolumn{8}{|c|}{ Luiz Eduardo Magalhães } & \multicolumn{8}{|c|}{ Vitória da Conquista } \\
\hline & \multicolumn{6}{|c|}{ Estrato da Planta } & \multicolumn{2}{|c|}{ Solo } & \multicolumn{6}{|c|}{ Estratos da Planta } & \multicolumn{2}{|c|}{ Solo } \\
\hline & \multicolumn{2}{|c|}{ Superior } & \multicolumn{2}{|c|}{ Médio } & \multicolumn{2}{|c|}{ Inferior } & \multirow[b]{2}{*}{$\mathbf{N}^{\mathbf{o}}$} & \multicolumn{3}{|c|}{ Superior } & \multicolumn{2}{|c|}{ Médio } & \multicolumn{2}{|c|}{ Inferior } & \multirow[b]{2}{*}{$\mathbf{N}^{\mathbf{o}}$} & \multirow[b]{2}{*}{$\%$} \\
\hline & $\mathbf{N}^{\mathbf{o}}$ & $\%$ & $\mathbf{N}^{\mathbf{o}}$ & $\%$ & $\mathbf{N}^{\mathbf{o}}$ & $\%$ & & $\%$ & $\mathbf{N}^{\mathbf{o}}$ & $\%$ & $\mathbf{N}^{\mathbf{o}}$ & $\%$ & $\mathbf{N}^{\mathbf{o}}$ & $\%$ & & \\
\hline Neochrysocharis coffeae & 89 & 81 & 31 & 72 & 29 & 56 & 52 & 85 & 26 & 17 & 13 & 15 & 17 & 15 & 22 & 28 \\
\hline Closterocerus coffeellae & 10 & 9 & 3 & 7 & 10 & 19 & 3 & 4,9 & 32 & 21 & 13 & 15 & 12 & 10 & 12 & 15 \\
\hline Stiropius sp.1 & 0 & 0 & 0 & 0 & 0 & 0 & 1 & 1,6 & 47 & 31 & 24 & 27 & 29 & 25 & 26 & 33 \\
\hline Stiropius sp. 2 & 0 & 0 & 0 & 0 & 0 & 0 & 1 & 1,6 & 4 & 2,6 & 13 & 15 & 19 & 17 & 0 & 0 \\
\hline Horismenus aeneicollis & 9 & 8 & 9 & 21 & 6 & 12 & 3 & 4,9 & 39 & 25 & 23 & 26 & 37 & 32 & 16 & 20 \\
\hline Cirrospilus neotropicus & 2 & 2 & 0 & 0 & 7 & 13 & 1 & 1,6 & 5 & 3,3 & 2 & 2 & 1 & 1 & 3 & 4 \\
\hline Total & 110 & & 43 & & 52 & & 61 & & 153 & & 88 & & 115 & & 79 & \\
\hline
\end{tabular}


condições de Vitória da Conquista, onde os braconídeos foram dominantes. Esses emergem das crisálidas, parasitando, provavelmente, lagartas em estádios finais de desenvolvimento. A distribuição do parasitismo, de modo geral, é coerente com a distribuição espacial do bichomineiro na planta de café, como verificado por Melo (2005), para as condições de Luiz Eduardo Magalhães, onde as minas se concentram no estrato superior da planta, com diferenças significativas em relação aos outros dois estratos $(\mathrm{p}<0,05)$. Para Vitória da Conquista, constatou-se número significativamente superior de crisálidas no terço inferior $(\mathrm{p}<0,5)$.

Do ponto de vista de programas de controle biológico do bicho-mineiro com parasitóides, para o Estado da Bahia, as espécies $H$. aeneicollis, $N$. coffeae e Stiropius sp.1 para as condições de Vitória da Conquista e $N$. coffeae para as condições de Luiz Eduardo Magalhães, pois se mostraram mais adaptadas e ocorreram na maior parte do ano. Dessa forma, investimentos em pesquisas visando a definição de estratégias de manutenção, criação e futuras liberações, poderiam ser direcionados a essas três espécies. Para avaliação da fauna de parasitóides do bicho-mineiro, recomenda-se amostragens nos três estratos da planta, ou, em caso de limitação operacional, nos estratos superior e inferior. Finalmente, ressalta-se a necessidade de estudos sobre comportamento de parasitismo dos braconídeos e eulofídeos, visando uma melhor compreensão das interações bitróficas.

\section{CONCLUSÕES}

São registradas, para as condições dos municípios de Vitória da Conquista e Luiz Eduardo Magalhães, as seguintes espécies de parasitóides do bicho-mineiro: Cirrospilus neotropicus, Closteroscerus coffeellae, Horismenus aeneicollis, Neochrysocharis coffeae, Stiropius sp.1 e Stiropius sp.2;

As espécies Horismenus aeneicollis, Closteroscerus coffeellae, Neochrysocharis coffeae e Stiropius sp.1 são predominantes nas condições de Vitória da Conquista;

O parasitóide Neochrysocharis coffeae é predominante nas condições de Luiz Eduardo Magalhães.

\section{AGRADECIMENTOS}

À Coordenação de Aperfeiçoamento do Pessoal de Nível Superior (CAPES), à Universidade Estadual do Sudoeste da Bahia - UESB e ao Consórcio Brasileiro de Pesquisas e Desenvolvimento do Café. Ao Dr. Valmir A. Costa (Instituto Biológico de Campinas), pelo auxílio na identificação dos parasitóides.

\section{REFERÊNCIAS BIBLIOGRÁFICAS}

AVILÉS, D. P. Avaliação das populações do bicho-mineiro do cafeeiro Perileucoptera coffeella (Lepidoptera: Lyonitiidae) e de seus parasitóides e predadores: metodologia de estudo e flutuação populacional. 1991. 126 f. Dissertação (Mestrado em Entomologia) - Universidade Federal de Viçosa, Viçosa, 1991.

ATIQUE, C. C.; FERREIRA, M. I. S.; COSTA, G. F. A. Aspectos biológicos dos Braconidae (Hymenoptera) parasitas de Perileucoptera coffeella, bicho-mineiro do cafeeiro. In: CONGRESSO BRASILEIRO DE PESQUISAS CAFEEIRAS, 9., 1981, São Lourenço. Anais... Rio de Janeiro: IBC/GERCA, 1981. p. 96-99.

CARNEIRO FILHO, F.; GUIMARÃES, P. M. Ocorrência de microhimenópteros parasitos de Leucoptera coffeella (GuérinMèneville, 1842) em três regiões do Estado do Paraná. In: CONGRESSO BRASILEIRO DE PESQUISAS CAFEEIRAS, 11., 1984, Londrina. Anais... Londrina: [s.n.], 1984. p. 115-116.

GALHARDO-COVAS, F. Augmentation of Mirax insularis Musebeck. alternative for population control of the coffee leaf miner, Leucoptera coffeella Guérin-Mèneville, in Puerto Rico. Journal Agriculture Puerto Rico, [S.1.], v. 76, n. 2, p. 43-54, 1992.

MELO, T. L. Flutuação populacional, predação e parasitismo do bicho-mineiro Leucoptera coffeella (Guérin-Méneville e Perrotet, 1842) ( Lepidoptera: Lyonetiidae) em duas regiões cafeeiras do Estado da Bahia. 2005. 134 f.Dissertação (Mestrado em Agronomia) - Universidade Estadual do Sudoeste da Bahia, Vitória da Conquista, 2005.

MENDES, L. O. T. Os parasitas do "bicho-mineiro das folhas de café". Revista do Instituto de Café, [S.1.], v. 26, n. 155, p. 6-11, 1940.

PARRA, J. R. P.; GONÇALVES, W.; GRAVENA, S.; MARCONATO, A. R. Parasitos e predadores do bichomineiro Perileucoptera coffeella (Guérin-Mèneville, 1842) em São Paulo. Anais da Sociedade Entomológica do Brasil, Londrina, v. 6, n. 1, p. 138-143, 1977.

PARRA, J. R. P.; GONÇALVES, W.; PRECETTI, A. A. C. M. Flutuação populacional de parasitos e predadores de Perileucoptera coffeella (Guérin- Meneville, 1842) em três localidades do Estado de São Paulo. Turrialba, San José, v. 4, p. 357-364, 1981. 
PERIOTO, N. W.; LARA, R. I. R.; SELEGATTO, A.; LUCIANO, E. S. Himenópteros parasitóides (Insecta, Hymenoptera) coletados em cultura de café Coffea arabica L. (Rubiaceae) em Ribeirão Preto, SP, Brasil. Arquivos do Instituto Biológico, São Paulo, v. 71, n. 1, p. 41-44, 2004.

RAIJ, B. V. Produção integrada de café. O Agronômico, Campinas, v. 55, n. 2, p. 14-15, 2003.

REIS JÚNIOR, R. Interferência entre vespas e parasitóides de Leucoptera coffeella (Guérin-Mèneville) (Lepidoptera: Lyonetiidae). 1999. 38 f. Dissertação (Mestrado em Entomologia) - Universidade Federal de Viçosa, Viçosa, 1999.

SILVA, E. J. E.; LOECK, A. E. Ocorrência de formigas domiciliares (Hymenoptera: Formicidae) em Pelotas, RS. Revista Brasileira de Agrociência, [S.1.], v. 5, n. 3, p. 220224, set./dez. 1999.
SILVEIRA NETO, S.; NAKANO, O.; BARBIN, D.; VILLANOVA, N. A. Manual de ecologia dos insetos. São Paulo: Agronômica Ceres, 1976. 419 p.

SOUZA, J. C. de. Levantamento, identificação e eficiência dos parasitos e predadores do "bicho-mineiro" das folhas do cafeeiro Perileucoptera coffeella (Guérin-Mèneville, 1842) (Lepidoptera: Lyonetiidae) no Estado de Minas Gerais. 1979. 90 f. Dissertação (Mestrado em Entomologia) - Escola Superior de Agricultura "Luiz de Queiroz", Universidade de São Paulo, Piracicaba, 1979.

SOUZA, J. C.; REIS, P. R. Pragas do cafeeiro: reconhecimento e controle. Viçosa: CTP, 2000. 54 p.

TOZATTI, G.; GRAVENA, S. Fatores naturais de mortalidade de Perileucoptera coffeella, Guérin-Mènveille (Lepidoptera - Lyonetiidae), em café, Jaboticabal. Científica, São Paulo, v. 16, n. 2, p. 179-187, 1988. 